\title{
Article
}

\section{Dexamethasone Induces Changes in Osteogenic Differentiation of Human Mesenchymal Stromal Cells via SOX9 and PPARG, but Not RUNX2}

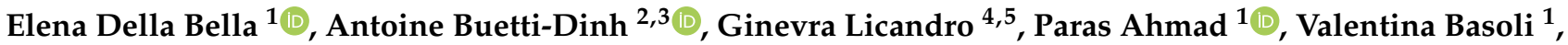 \\ Mauro Alini ${ }^{1}$ and Martin J. Stoddart $1,6, *$ (D)
}

1 AO Research Institute Davos, 7270 Davos Platz, Switzerland; elena.dellabella@aofoundation.org (E.D.B.); docparas2017@gmail.com (P.A.); valentina.basoli@aofoundation.org (V.B.); mauro.alini@aofoundation.org (M.A.)

2 Laboratory of applied microbiology (LMA), Department of Environment, Constructions and Design (DACD), University of Applied Sciences of Southern Switzerland (SUPSI), 6500 Bellinzona, Switzerland; antoine.buetti@supsi.ch

3 Swiss Institute of Bioinformatics, Quartier Sorge-Batiment Genopode, 1015 Lausanne, Switzerland

4 Dalle Molle Institute for Artificial Intelligence (IDSIA), University of Italian Switzerland (USI), 6928 Manno, Switzerland; ginevra.licandro@idsia.ch

5 University of Applied Science and Art of Southern Switzerland (SUPSI), 6928 Manno, Switzerland

6 Department of Orthopedics and Trauma Surgery, Medical Center-Albert-Ludwigs-University of Freiburg, Faculty of Medicine, Albert-Ludwigs-University of Freiburg, 79106 Freiburg, Germany

* Correspondence: martin.stoddart@aofoundation.org; Tel.: +41-81-414-24-48

check for updates

Citation: Della Bella, E.; Buetti-Dinh A.; Licandro, G.; Ahmad, P.; Basoli, V.; Alini, M.; Stoddart, M.J.

Dexamethasone Induces Changes in Osteogenic Differentiation of Human Mesenchymal Stromal Cells via SOX9 and PPARG, but Not RUNX2. Int. J. Mol. Sci. 2021, 22, 4785. https:// doi.org/10.3390/ijms22094785

Academic Editor: Giacomina Brunetti

Received: 19 March 2021

Accepted: 28 April 2021

Published: 30 April 2021

Publisher's Note: MDPI stays neutra with regard to jurisdictional claims in published maps and institutional affiliations.

Copyright: (c) 2021 by the authors. Licensee MDPI, Basel, Switzerland. This article is an open access article distributed under the terms and conditions of the Creative Commons Attribution (CC BY) license (https:// creativecommons.org/licenses/by/ $4.0 /)$.
Abstract: Despite the huge body of research on osteogenic differentiation and bone tissue engineering, the translation potential of in vitro results still does not match the effort employed. One reason might be that the protocols used for in vitro research have inherent pitfalls. The synthetic glucocorticoid dexamethasone is commonly used in protocols for trilineage differentiation of human bone marrow mesenchymal stromal cells (hBMSCs). However, in the case of osteogenic commitment, dexamethasone has the main pitfall of inhibiting terminal osteoblast differentiation, and its pro-adipogenic effect is well known. In this work, we aimed to clarify the role of dexamethasone in the osteogenesis of hBMSCs, with a particular focus on off-target differentiation. The results showed that dexamethasone does induce osteogenic differentiation by inhibiting SOX9 expression, but not directly through RUNX2 upregulation as it is commonly thought. Rather, PPARG is concomitantly and strongly upregulated, leading to the formation of adipocyte-like cells within osteogenic cultures. Limiting the exposure to dexamethasone to the first week of differentiation did not affect the mineralization potential. Gene expression levels of RUNX2, SOX9, and PPARG were simulated using approximate Bayesian computation based on a simplified theoretical model, which was able to reproduce the observed experimental trends but with a different range of responses, indicating that other factors should be integrated to fully understand how dexamethasone influences cell fate. In summary, this work provides evidence that current in vitro differentiation protocols based on dexamethasone do not represent a good model, and further research is warranted in this field.

Keywords: Osteogenesis; glucocorticoids; transcription factors; MSC; gene expression; approximate Bayesian computation (ABC)

\section{Introduction}

Bone is the second most transplanted tissue after blood [1]. Autologous grafting is the gold-standard treatment for bone replacement when bone healing capability is impaired. Nonetheless, its application can be limited by several factors and complications [2]. The use of bone substitutes therefore has a great clinical need, but despite the research and effort there is still a poor translational potential of in vitro results to in vivo studies in the 
field of biomaterial testing for bone regeneration [3]. This urges the need to go back to the bench and reconsider the way in which osteogenic differentiation and bone are investigated in vitro.

Glucocorticoids are powerful immunomodulatory and anti-inflammatory drugs, widely prescribed for the treatment of idiopathic conditions with a strong inflammatory component such as chronic obstructive pulmonary disease, rheumatoid arthritis, inflammatory bowel disease, and autoimmune disorders [4]. The long-term use of such drugs, however, comes at the expense of serious side effects, such as osteoporosis $[5,6]$ and osteonecrosis [7,8]. Glucocorticoids exert profound effects on bone and are crucial for human osteoblast differentiation, along with the formation of the extracellular matrix [9]. Moreover, they play a vital role in controlling skeletal development and maintaining healthy bone. Despite clear induction of osteogenesis by glucocorticoids in vitro, they are still considered to be negative regulators of osteogenesis [10]. Glucocorticoids become most harmful to bone at supra-physiological levels and lead to skeletal fragility [11]. Although all bone cells are affected by high levels of glucocorticoids, rodent models reliably demonstrate that the essential targets of glucocorticoids in the skeleton are osteocytes and osteoblasts [11]. In particular, the viability of the former is predominantly associated with bone strength and is readily compromised by glucocorticoids at supraphysiological levels [12]. This mechanism of action has eventually led to the investigation of novel drug intervention approaches with osteoblast-targeted agents. Emerging osteoanabolic strategies are specifically suited to offset the detrimental effects of therapeutic glucocorticoids on the structural and molecular levels [13].

The synthetic glucocorticoid dexamethasone (dex) is commonly used in vitro in trilineage differentiation protocols for mesenchymal stromal cells (MSCs). In bone research, dex has been used for osteogenic differentiation of bone-marrow-derived MSCs (BMSCs) for almost 30 years [14], and differentiation cocktails containing at least $10 \mathrm{nM}$ dex, together with ascorbic acid and a source of phosphate groups, are the standard in the field [15-17]. Dex has been reported to increase the expression of $R U N X 2[15,18]$, which is considered the master regulator of osteogenic differentiation [19]. However, the precise mechanism by which dex induces osteogenic differentiation might be cell-type-dependent, as in cells from dental follicle or dental pulp it seems to rely on ZBTB16 but not on RUNX2 [20,21]. It has also been reported that an initial burst of high-dose dex (100 nM for the first week of differentiation) could improve osteogenesis and reduce variability between donors [15,22]. Even though the use of dex seems to be necessary to address BMSCs to an osteogenic lineage and to achieve a good level of mineralization and differentiation in vitro [23], it has the main pitfall of repressing osteocalcin expression and inhibiting terminal differentiation $[24,25]$. One main mechanism of glucocorticoid-mediated osteocalcin transcriptional repression is achieved through an Egr/Krox site [25]. More recently, it has been observed that dex regulates histone deacetylase 6 (HDAC6) expression, and that a glucocorticoid receptor (GR)-HDAC6 repressor complex occupies both proximal and distal regions of the osteocalcin promoter, leading to the inhibition of osteocalcin transcription [24].

The glucocorticoid receptor pathway is complex and wide, and we previously demonstrated an effect of dex on human BMSC non-coding RNA expression which was independent of the differentiation status [26]. Among other side effects, dex is known to possess a pro-adipogenic effect, indeed it was demonstrated to promote adipose tissue at the expense of bone formation in bone marrow [10]. In murine cells, it was observed that dex may lead to heterogeneous osteogenic/adipogenic differentiation [27]. Notably, it was also suggested that osteoblasts with a high PPARG expression may switch fate and become adipocytes [28].

For these reasons, the aim of this study was to clarify the role of dex during the osteogenic differentiation of human BMSCs (hBMSCs), with a particular focus on simultaneous adipogenic commitment. The effect of dex on the three main lineage-specific transcription factors for hBMSCs, namely RUNX2, SOX9, and PPARG [29], was studied, 
with a main focus on early differentiation (day 7), which is when key lineage decisions are made $[30,31]$.

\section{Results}

\subsection{Dose-Dependent Effect of Dex on Differentiation}

We first analyzed the effect of dex on osteogenic differentiation, with a particular focus on mineralization and on the formation of lipid-droplet-containing cells. After 21 days in an osteogenic-supporting environment (i.e., containing ascorbic acid and glycerol 2-phosphate), the presence of dex showed two separate effects. First, it increased calcium deposition, as expected (Figure 1A,B). In one donor, the presence of an osteogenic environment alone was sufficient alone to boost mineralization, which was not improved further by dex. The RUNX2/SOX9 ratio at day 7 was shown to positively correlate with Alizarin Red staining at day 21, with a suggested dex dose-dependency (Figure 1C). In parallel, osteogenic cultures from the same donors were stained with Oil Red $\mathrm{O}$ to determine the presence of lipid droplets. The results showed that the increase in dex concentration concurrently led to an increase in the presence of pre-adipocyte-like cells, in particular for the $100 \mathrm{nM}$ dose (Figure 1D,E). Analogously to the correlation between RUNX2/SOX9 and mineralization, PPARG expression at day 7 positively correlated with the $\%$ area covered by Oil Red-stained cells at day 21 (Figure 1F).

\section{A)}

d176

d200

d205

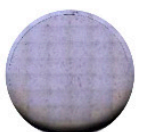

.
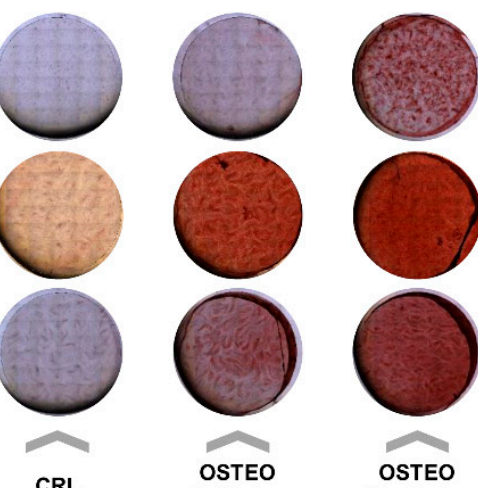

CRL

D)
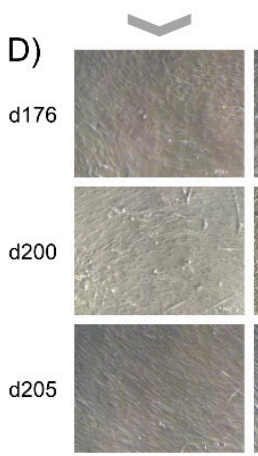
$0 \mathrm{nM}$ dex
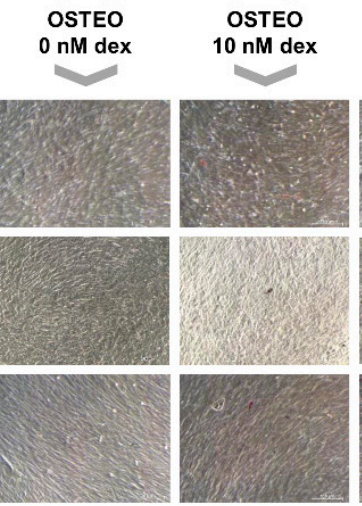

B)

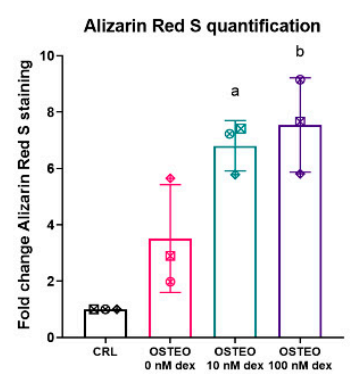

E)

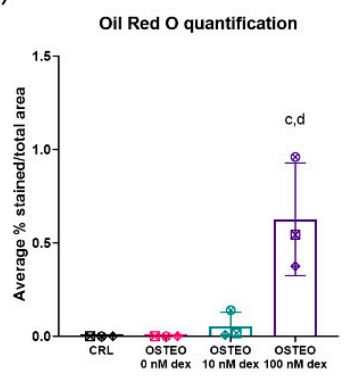

C)

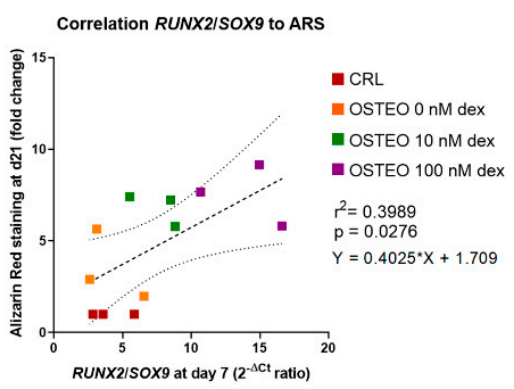

F)

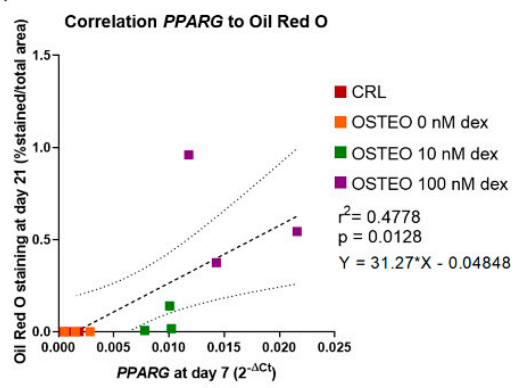

Figure 1. Differentiation potential of hBMSCs from three donors, using different concentrations of dex (0, 10, 100 nM). (A) Alizarin Red S (ARS) staining after 21 days of osteogenic differentiation, showing an increase in calcium incorporation. Images represent mosaics of pictures, transmitted light, and brightfield images. The diameter of Thermanox coverslips, which were fully pictured, was $22 \mathrm{~mm}$. (B) ARS staining quantification was performed via elution of the stain from the cultures and the subsequent spectrophotometric determination of optical density. For each donor (represented by a different symbol), the results are expressed as a fold-change in intensity of staining compared to the undifferentiated controls. Summary of results from a two-way Analysis of Variance (ANOVA) with Tukey post-hoc test. a: $p<0.05$; Osteo $10 \mathrm{nM}$ dex vs. CRL. b: $p<0.01$; Osteo 100 nM dex vs. CRL. (C) RUNX2/SOX9 ratio correlation to ARS. Pearson's correlation was calculated to correlate the RUNX2/SOX9 ratio ( $2^{-\Delta C t}$ ratio) at day 7 with the amount of Alizarin Red staining at day 21 , 
expressed as a fold-change in comparison to the undifferentiated control. The correlation was found to be significant, with a $p$-value of 0.0276 , pointing towards a higher calcium deposition with higher RUNX2/SOX9 levels. The $\mathrm{R}^{2}$ value obtained (0.39898) suggests other factors were involved in the process, which influenced the linear correlation. The dashed line represents the line of best fit, of which the equation is reported. The dotted lines represent the $95 \%$ confidence interval. (D) Oil Red O staining after 21 days of osteogenic differentiation, showing a substantial increase in adipogenic differentiation with $100 \mathrm{nM}$ dex. Scale bar $=200 \mu \mathrm{m}$ (bottom-right image). (E) Oil Red O staining quantification performed via image analysis with ImageJ. The results are expressed as the average \% stained area of the total area of the field. Each determination is the average of 10 different fields for each condition. Summary of results from a two-way ANOVA with the Tukey post-hoc test. c: $p<0.01$; Osteo $100 \mathrm{nM}$ dex vs. CRL and vs. Osteo $0 \mathrm{nM}$ dex. d: $p<0.05$; Osteo $100 \mathrm{nM}$ dex vs. Osteo $10 \mathrm{nM}$ dex. (F) Correlation of PPARG expression at day 7 with Oil Red $\mathrm{O}$ staining at day 21. The results show a positive correlation between the two factors. The dashed line represents the line of best fit, of which the equation is reported. The dotted lines represent the $95 \%$ confidence interval.

The gene expression levels of key transcription factors (Figure 2) and late markers of differentiation (Figure 3) were analyzed. The results showed that RUNX2 was not significantly regulated by dex. However, a trend towards a downregulation of SOX9 with increasing concentrations of dex was observed, which resulted in a significantly higher RUNX2/SOX9 ratio with $100 \mathrm{nM}$. In line with the identification of adipocyte-like cells within the osteogenic cultures, the levels of PPARG were considerably upregulated by dex in a dose-dependent manner, in particular at day 7. Other transcription factors with an involvement in osteogenic differentiation showed no significant change in expression, with a trend toward higher levels of DLX5 and SP7 with $10 \mathrm{nM}$ dex at day 7 only, which might reflect a higher activity in early osteogenic commitment (Figure 2).
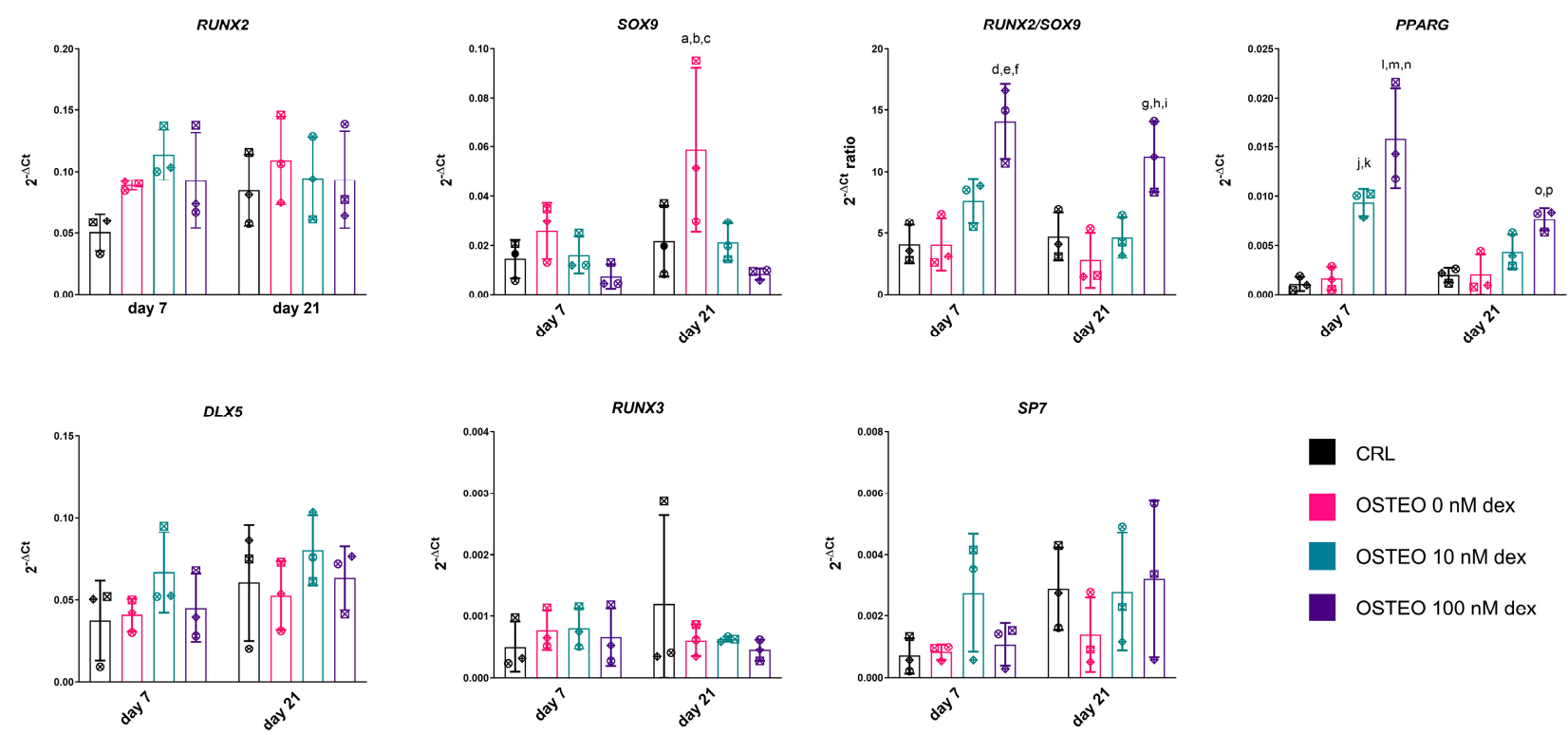

Figure 2. Expression of genes encoding for transcription factors with a known influence on osteogenic or adipogenic differentiation. Day 7 and day 21 data in undifferentiated controls and osteogenic commitment with 0, 10, and 100 nM dex are reported. Summary of results from a two-way ANOVA with the Tukey post-hoc test. SOX9. a: $p<0.05 ;$ Osteo $0 \mathrm{nM}$ dex vs. CRL at day 21. b: $p<0.05$; Osteo $0 \mathrm{nM}$ dex vs. Osteo $10 \mathrm{nM}$ dex at day 21. c: $p<0.01$; Osteo $0 \mathrm{nM}$ dex vs. Osteo $100 \mathrm{nM}$ dex at day 21. RUNX2/SOX9. d: $p<0.001$; Osteo $100 \mathrm{nM}$ dex vs. CRL at day 7. e: $p<0.001$; Osteo $100 \mathrm{nM}$ dex vs. Osteo $0 \mathrm{nM}$ dex at day 7. f: $p<0.05$; Osteo $100 \mathrm{nM}$ dex vs. Osteo $10 \mathrm{nM}$ dex at day 7. g: $p<0.05$; Osteo $100 \mathrm{nM}$ dex vs. CRL at day 21. h: $p<0.01$; Osteo $100 \mathrm{nM}$ dex vs. Osteo $0 \mathrm{nM}$ dex at day 21. i: $p<0.05$; Osteo $100 \mathrm{nM}$ dex vs. Osteo $10 \mathrm{nM}$ dex at day 21. PPARG. j: $p<0.01$; Osteo $10 \mathrm{nM}$ dex vs. CRL at day 7. k: $p<0.01$; Osteo $10 \mathrm{nM}$ dex vs. Osteo $0 \mathrm{nM}$ dex at day 7. 1: $p<0.0001$; Osteo $100 \mathrm{nM}$ dex vs. CRL at day 7. m: $p<0.0001$; Osteo $100 \mathrm{nM}$ dex vs. Osteo $0 \mathrm{nM}$ dex at day 7. n: $p<0.05 ;$ Osteo $100 \mathrm{nM}$ dex vs. Osteo $10 \mathrm{nM}$ dex at day 7. o: $p<0.05$; Osteo $100 \mathrm{nM}$ dex vs. CRL dex at day 21. p: $p<0.05$; Osteo $100 \mathrm{nM}$ dex vs. Osteo $0 \mathrm{nM}$ dex at day 21. RUNX2, DLX5, RUNX3, SP7: n.s. Each symbol corresponds to a unique donor, whereas colors indicate a different group, as indicated in the legend. 

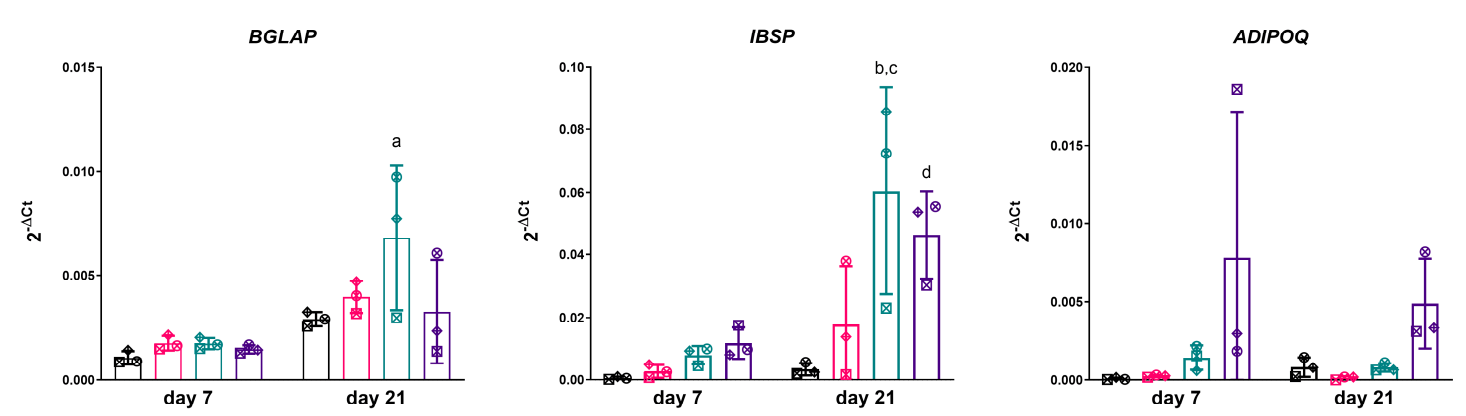

CRL

OSTEO $0 \mathrm{nM}$ dex

OSTEO $10 \mathrm{nM}$ dex

OSTEO $100 \mathrm{nM}$ dex

Figure 3. Expression of genes encoding for late markers of differentiation, i.e., osteocalcin (BGLAP) and bone sialoprotein $(I B S P)$ for osteogenic differentiation, and adiponectin $(A D I P O Q)$ as an adipocyte marker. Summary of statistical analysis with a two-way ANOVA with the Tukey post-hoc test. BGLAP. a: $p<0.05$; Osteo $10 \mathrm{nM}$ dex vs. CRL, day 21. IBSP. b: $p<0.001$; Osteo $10 \mathrm{nM}$ dex vs. CRL, day 21. c: $p<0.05$; Osteo $10 \mathrm{nM}$ dex vs. Osteo $0 \mathrm{nM}$ dex, day 21. d: $p<0.05$; Osteo $100 \mathrm{nM}$ dex vs. CRL, day 21. ADIPOQ: n.s. Each symbol corresponds to a unique donor, whereas colors indicate a different group, as indicated in the legend.

The expression of late markers of differentiation revealed a peak of BGLAP and IBSP expression with $10 \mathrm{nM}$ dex at day 21, which was reduced with the higher dose of glucocorticoid. Interestingly, although not significantly, adiponectin appeared to be more expressed in $100 \mathrm{nM}$ dex (Figure 3).

\subsection{Effect of Dex on RUNX2, SOX9, and PPARG Expression}

The early expression of RUNX2, SOX9, and PPARG was studied in a larger number of donors (timepoint: day $7, \mathrm{n}=8$ ). Cultures were also treated for comparison with the same concentrations of (+)-ZK 216348, a synthetic glucocorticoid which shares the transrepressional activity of dex on glucocorticoid receptor but does not stimulate the transactivation pathways [32]. The results confirmed that dex had no effect on the expression of RUNX2, while strongly inhibiting SOX9 and upregulating PPARG levels in a dose-dependent manner (Figure 4). The trend for SOX9 and PPARG gene expression was consistent among donors. SOX9 was upregulated in the presence of ascorbic acid and glycerol 2-phosphate alone, and the addition of $10 \mathrm{nM}$ and $100 \mathrm{nM}$ dex strongly suppressed SOX9 expression. On the contrary, PPARG levels were unaltered when ascorbic acid and glycerol 2-phosphate were added, but the presence of dex resulted in a dose-dependent increase in its expression. (+)-ZK 216348 showed no effect on gene expression of either RUNX2, SOX9, or PPARG, although a non-significant trend in SOX9 downregulation and PPARG upregulation can be observed when using higher concentrations of this glucocorticoid receptor agonist. 

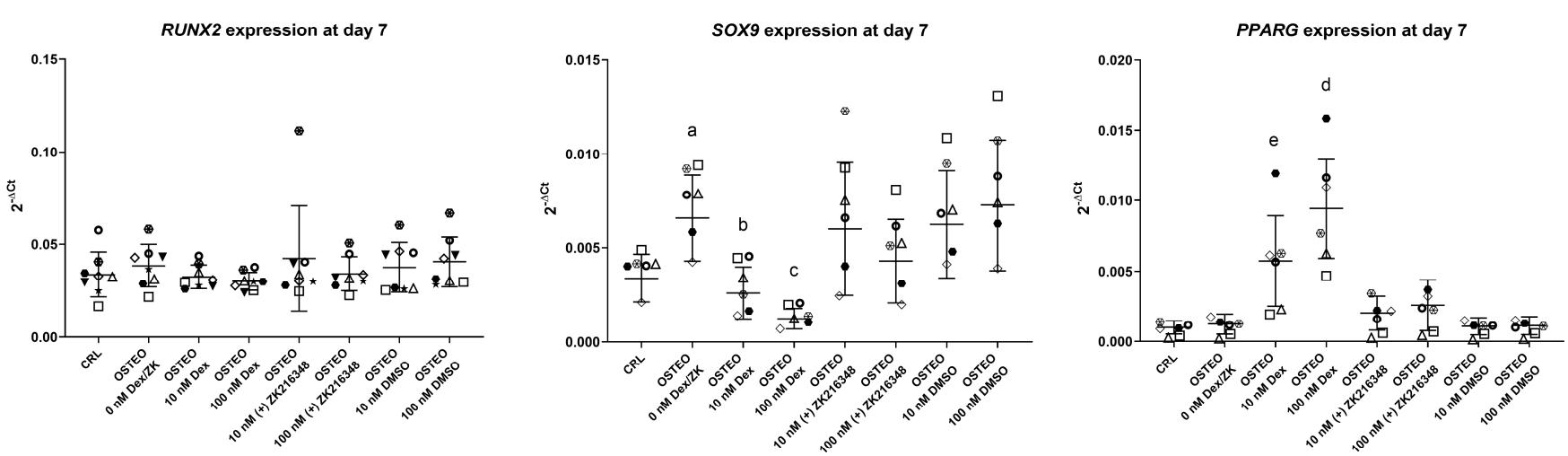

Figure 4. Effect of dex and (+)-ZK 216348 on the expression of RUNX2, SOX9, and PPARG at day 7. N = 8 donors (each represented by a unique symbol). Dimethyl sulfoxide (DMSO) groups were also used as vehicle controls for (+)-ZK 216348. Summary of statistical analysis. RUNX2. n.s. SOX9. a: $p<0.01$ OSTEO $0 \mathrm{nM}$ dex/ZK vs CRL, dex groups, $100 \mathrm{nM}(+)-Z K$ 216348. b: $p<0.01$ OSTEO $10 \mathrm{nM}$ dex vs. $0 \mathrm{nM}$ dex; $p<0.05$ OSTEO $10 \mathrm{nM}$ dex vs. $100 \mathrm{nM}$ dex, $100 \mathrm{nM}$ (+)-ZK 216348 and DMSO groups. c: $p<0.01$ OSTEO $100 \mathrm{nM}$ dex vs. CRL, $0 \mathrm{nM}$ and DMSO groups; $p<0.05$ OSTEO $100 \mathrm{nM}$ dex vs. $10 \mathrm{nM}$ dex and (+)-ZK 216348 groups. PPARG. d: $p<0.01$; OSTEO $100 \mathrm{nM}$ dex vs. all groups. e: $p<0.05$; OSTEO $10 \mathrm{nM}$ dex vs. all groups ( $p<0.01$ vs. $100 \mathrm{nM}$ dex).

\subsection{Two-Stage Osteogenic Differentiation}

Osteogenic differentiation was further induced using hBMSCs from four additional donors. A two-stage protocol was employed, divided into week 1 and week $2+3$. Simply, the protocols differed in the concentration of dex used (Table 1). The Osteo $100 \mathrm{nM}$ group for 21 days was not included at this point. The results showed that reducing the concentrations of dex after the first week of induction did not impair the mineralization potential, as depicted in Figure 5. Indeed, Alizarin Red staining levels remained at comparable levels to when $10 \mathrm{nM}$ dex were maintained for the whole 3 weeks of differentiation. The use of a high dose $(100 \mathrm{nM})$ for the first week did not significantly improve calcium deposition. Interestingly, the correlation between RUNX2/SOX9 ratio at day 7 and Alizarin Red staining at day 21 was maintained even when the differentiation medium was changed after the first week. The expression of late osteogenic markers was reduced in group \#5 and improved when dex was completely withdrawn after the first week (group \#6), though the results do not reach statistical significance.

Table 1. Scheme of the two-stage protocols for osteogenic differentiation. The osteogenic differentiation medium (OSTEO) contains $5 \mathrm{mM} \beta$-glycerol phosphate and $50 \mu \mathrm{g} / \mathrm{mL}$ ascorbic acid 2-phosphate, with the indicated amount of dex. CRL medium refers to the growth medium containing DMEM, $10 \%$ FBS, and penicillin/streptomycin only.

\begin{tabular}{ccc}
\hline Group & Week 1 & Week 2 + 3 \\
\hline$\# 1$ & CRL medium & CRL medium \\
\hline$\# 2$ & OSTEO 10 $\mathrm{nM}$ dex & OSTEO 10 $\mathrm{nM}$ dex \\
\hline$\# 3$ & OSTEO 0 nM dex & OSTEO 0 nM dex \\
\hline$\# 4$ & OSTEO 10 $\mathrm{nM}$ dex & OSTEO 0 nM dex \\
\hline$\# 5$ & OSTEO 100 $\mathrm{nM}$ dex & OSTEO 10 nM dex \\
\hline$\# 6$ & OSTEO 100 $\mathrm{nM}$ dex & OSTEO 0 $\mathrm{nM}$ dex
\end{tabular}


A)

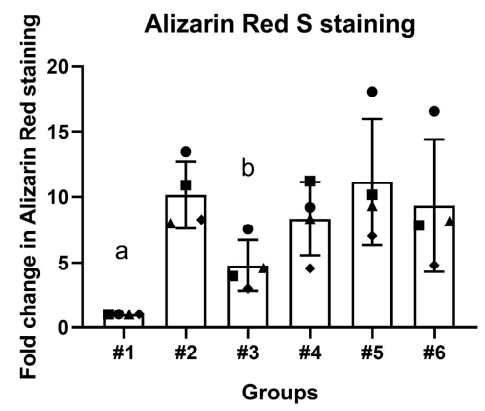

C)

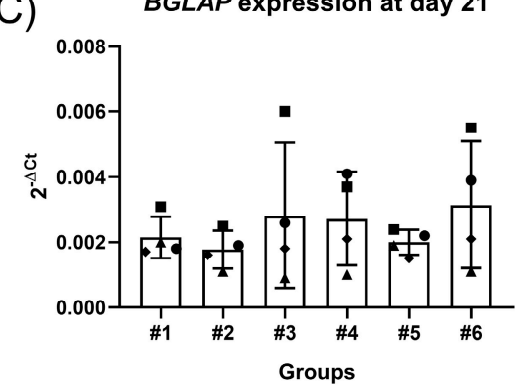

B)

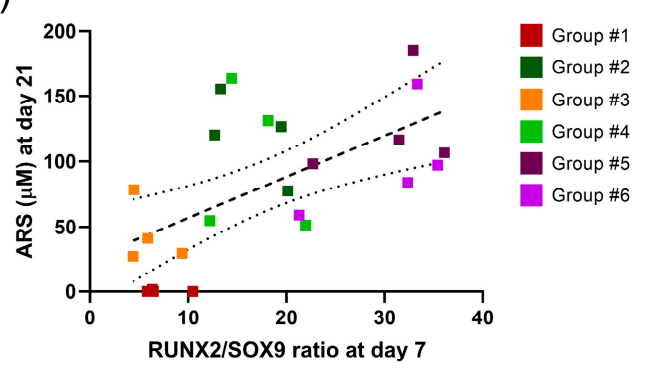

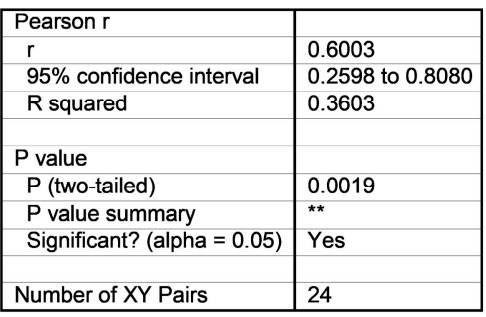

Numer of XY Pairs
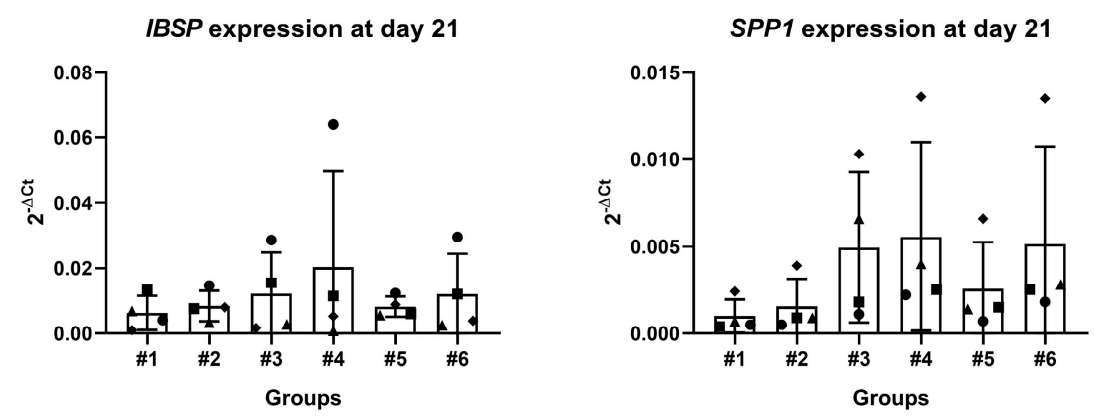

Figure 5. Osteogenic differentiation using different timings and concentration of dex. Each donor is represented by a unique symbol. (A) Alizarin Red staining quantification. For each donor, the results are expressed as a fold-change in the intensity of staining compared to the undifferentiated controls. a: $p<0.001$, \#1 vs. \#2; $p<0.01$, \#1 vs. \#4, $p<0.001$, \#1 vs. \#5; $p<0.01$, $\# 1$ vs. \#6. b: $p<0.05$, \#3 vs. 2 and \#3 vs. 5. (B) RUNX2/SOX9 ratio correlation to ARS. Pearson's correlation was calculated to correlate the RUNX2/SOX9 ratio ( $2^{-\Delta \mathrm{Ct}}$ ratio) at day 7 with amount of Alizarin Red staining at day 21, expressed as a fold-change in comparison to the undifferentiated control. The correlation was found to be significant, with a $p$-value of 0.0019. The dashed line represents the line of best fit, of which the equation is reported. The dotted lines represent the $95 \%$ confidence interval. (C) Gene expression level of late osteogenic markers osteocalcin (BGLAP), integrin-binding sialoprotein (IBSP) and osteopontin (SPP1).

\subsection{Expression Profiles of RUNX2, SOX9, and PPARG}

Data on RUNX2, SOX9, and PPARG expression were gathered for all the donors involved in this study (Figure 6A). The results from all the donors confirm that dex does not alter RUNX2 expression, at least at day 7, with upregulation and downregulation of $P P A R G$ and SOX9, respectively.

To assess differences in terms of gene expression levels between samples and similarities among donors' profiles, the relative transcription levels of the treated samples (osteogenic medium with different concentrations of dex) were normalized to the expression in the respective control group (Log2 of the fold change [Log2FC], see Supplementary Table S2), and the results were represented through hierarchical heatmaps (Figure 6B). Dex at both concentrations upregulated PPARG in all donors ( $\log 2 \mathrm{FC} \geq 1.47$ ); in the majority of the donors, upregulation was also measured in the osteogenic medium in the absence of dex but at a lower level, except for donors \#1, \#8, \#9, and \#12. On the contrary, for SOX9 a polarization to downregulation was observed in a dose-dependent manner, despite a tendency to be upregulated in the presence of osteogenic medium alone (Log2FC range (0.03-1.47)). Interestingly, donor \#13 showed almost no effect at $10 \mathrm{nM}$ dex $(\log 2 \mathrm{FC}=1.18)$ compared to osteogenic buffer alone $(\log 2 \mathrm{FC}=1.05)$. 

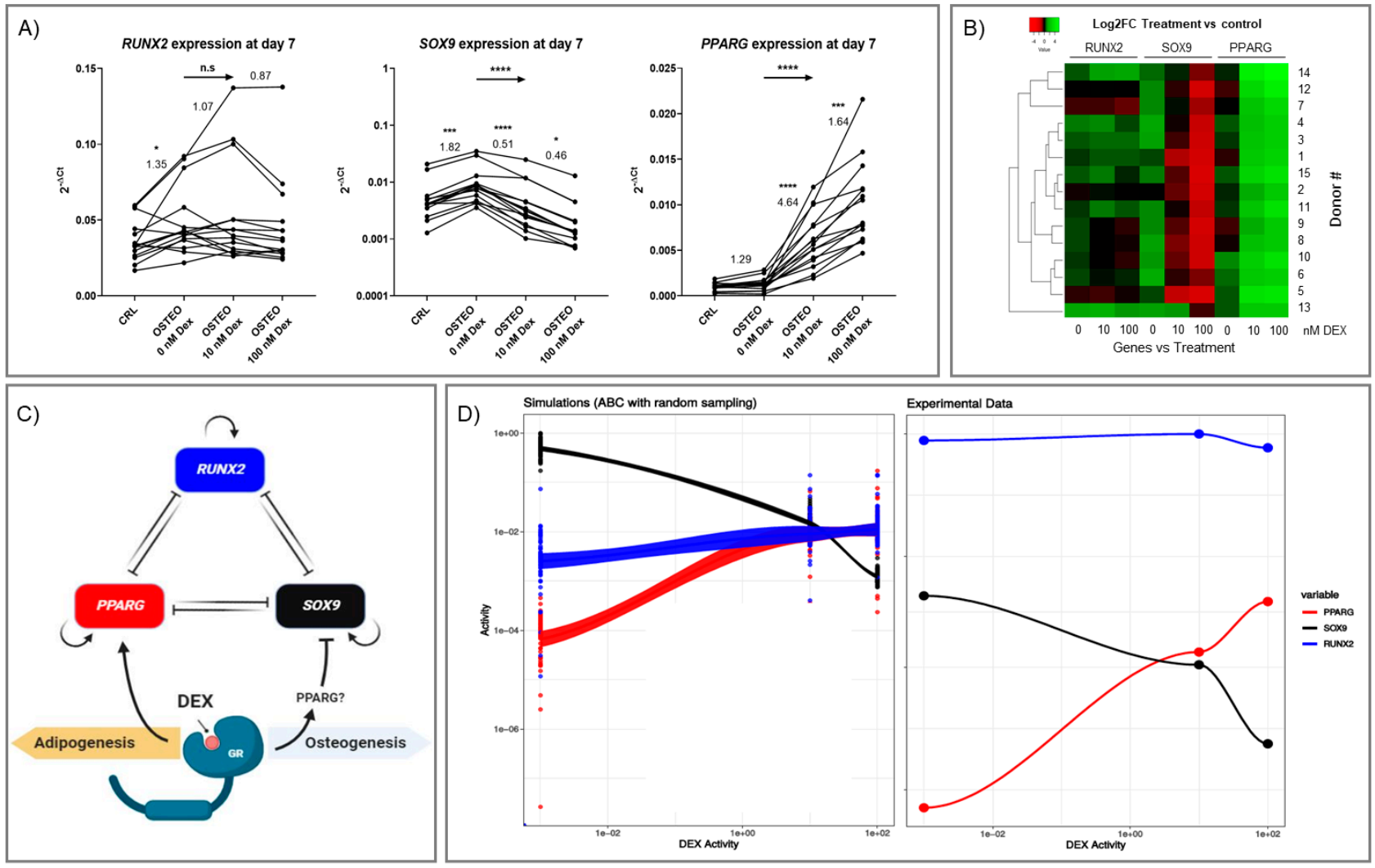

Figure 6. (A) Gene expression of RUNX2, SOX9, and PPARG at day 7 during osteogenic differentiation with increasing concentrations of dex. CRL: DMEM-LG, 10\% FBS, 1\% P/S. OSTEO 0 nM dex: OSTEO medium (DMEM-LG, 10\% FBS, 1\% $\mathrm{P} / \mathrm{S}, 50 \mu \mathrm{g} / \mathrm{mL}$ ascorbic acid 2-phosphate, $5 \mathrm{mM}$ beta-glycerol phosphate) with no dex added. OSTEO $10 \mathrm{nM}$ dex: OSTEO medium with $10 \mathrm{nM}$ dex. OSTEO $100 \mathrm{nM}$ dex: OSTEO medium with $100 \mathrm{nM}$ dex. $\mathrm{n}=15$ donors. The before-after graphs depict the expression of RUNX2, SOX9, and PPARG in each individual donor in the different conditions, expressed as $2^{-\triangle \mathrm{Ct}}$. A repeated measures one-way ANOVA with a post-hoc test for trends confirmed no effect of dex on RUNX2, whereas a dose-dependent effect of dex on SOX9 downregulation $\left.{ }^{* * * *}, p<0.0001\right)$ and PPARG upregulation $(* * * *, p<0.0001)$ was observed. The numbers above the lines connecting the conditions indicate the average ratio of expression between two different conditions: from left to right, OSTEO $0 \mathrm{nM}$ dex/CRL; OSTEO $10 \mathrm{nM}$ dex/OSTEO $0 \mathrm{nM}$ dex; OSTEO $100 \mathrm{nM}$ dex/OSTEO $10 \mathrm{nM}$ dex. The asterisks indicate if there is a difference between the two conditions compared; ${ }^{*} p<0.05$; ${ }^{* * *} p<0.001 ;{ }^{* * * *} p<0.0001$. The rightward arrows above the graphs summarize the results of the ANOVA test for linear trends in the left-to-right order: n.s., non-significant: ${ }^{* * * *} p<0.0001$ (SOX9 and PPARG show a dex dose-dependent change). (B) Gene expression profiling of RUNX2, SOX9, and PPARG in response to pro-osteogenic medium with different concentrations of $\operatorname{dex}(0 \mathrm{nM}, 10 \mathrm{nM}, 100 \mathrm{nM})$ in isolated hBMSCs. The data were visualized by a heat-map and the color legend shows the relative expression normalized to the expression level of the respective untreated condition for each gene (Log2FC). The color changes from red (downregulation) to green (upregulation). Clustering analysis indicates the similarities among transcription profiles of donors for the genes of interest. (C) Theoretical model of RUNX2-SOX9-PPARG interaction (Figure created with Biorender.com). The basic model was proposed by MacArthur et al. [33]. Based on our experimental results, the model was modified to account for the influence of dex-activated glucocorticoid receptor on gene expression. Dex seemed to exert its effect mainly through SOX9 and PPARG regulation. (D) Signaling model compared to experimentally measured activities of PPARG, SOX9, and RUNX2. ABC simulations (left) used to determine the parameters of the theoretical model from random sampling that best represent the experimentally measured activity values (right) of PPARG, SOX9, and RUNX2. Corridor lines represent the $\mathrm{R}$ stat_smooth confidence interval over the simulated data points.

As already observed, RUNX2 showed the most heterogeneous results among donors. As further emphasized in Figure S3, four main clusters of patients were present, with \#5 being most dissimilar to the other donors.

Based on the gene expression results, a simplified theoretical model of the interaction among transcription factors [33] was modified to take the effect of dex into ac- 
count (Figure 6C). The signaling network was used to perform steady-state simulations (Figure 6D, left) [34-36]. The activity profile of the in-silico network was then compared to the experimentally observed activities via approximate Bayesian computation (ABC) [37]. This allows the estimation of the physicochemical parameters underlying the network regulation in the present experimental context.

The simulations successfully captured the trend observed experimentally, indicating a $~ 50$-fold lower degradation rate for SOX9 as a hallmark of the current observations. However, the proportion of the relative response between RUNX2, SOX9, and PPARG appears to be different. Simulations predict RUNX2 to have a lower activity than what it is observed experimentally (Figure 6D).

$\mathrm{ABC}$ allowed us to estimate model parameter values that led steady-state simulations to match as well as possible with the results observed experimentally (Figure 6A). Figure S1 shows the uniformly random prior distribution of the parameters used as inputs in the $A B C$ procedure. $A B C$ then selected the parameter ranges for which the steady-state model matched the observed experimental values of Figure 6A (see also Figure S2).

The estimated decay rates of PPARG and RUNX2 indicated values in the central to upper range of the sampled space, supporting a relatively faster degradation than SOX9, which was restricted to a narrow range at low values (see Figure S2, first row).

The affinities of the inhibitory interaction between RUNX2, SOX9, and PPARG indicated selected values in the central range of the sampled parameters, with exceptions for the affinities of RUNX2 to SOX9 and of SOX9 to RUNX2, which were slightly skewed to a higher and a lower parameter range, respectively (Figure S2, second and third rows). This indicated that, in the mutual inhibitory interaction between RUNX2 and SOX9, SOX9 exerted a stronger effect on RUNX2 than the opposite reaction (as affinity here was defined as a dissociation binding constant - the smaller the value the stronger the molecular interaction).

Finally, ABC indicated a more important role for the strength of the SOX9 feedback loop compared to PPARG and RUNX2 feedbacks. SOX9 feedback more frequently assumed low values (strong feedback) in the simulations selected by $A B C$ to be matching the experimental results (Figure S2, last row).

\section{Discussion}

Despite the physiological regenerative capability of bone and the huge body of research on osteogenic differentiation, the potential for the translation of in vitro results is still quite limited [3] and to date there are no bone-related clinical tissue engineering applications approved that include a cellular component (as per April 2021, see https:/ / www.fda.gov/vaccines-bloodbiologics / cellular-gene-therapy-products / approved-cellular-and-gene-therapy-products for FDA approvals, accessed on 19 March 2021) [38]. One of the simplest explanations for this phenomenon is that there are some inherent issues with the experimental protocols that are employed to study in vitro osteogenesis. In this regard, it is striking that the majority of protocols for inducing osteogenic differentiation in bone-marrow-derived mesenchymal stromal cells relies on the use of dex. Even though it has a pro-osteogenic effect, the evidence from the clinical use of glucocorticoids is that it negatively affects bone tissue and vasculature health, with serious sequelae for the patient in the long term. [39] Combining these apparently contradictive effects with the presence of dex in differentiation protocols toward other classical mesenchymal lineages (chondro- and adipogenic), this raised the question of whether dex is the weakest link in the chain.

To answer this question, we started looking at the effect of increasing dex concentrations on pro-osteogenic cultures, focusing on gene expression and final differentiation outcomes. This work provides evidence for an off-target adipocytic differentiation in standard osteogenic cultures of hBMSCs. This effect is induced by dex, which strongly promotes PPARG expression in a dose-dependent manner. The presence of pre-adipocyte-like cells, even if it is limited, should warn us that the protocol currently in use needs to be reconsidered because it is not possible to control unwanted cell fate commitment. Even though our standard protocols use $10 \mathrm{nM}$ dex, which showed a more limited presence-but 
not the absence - of lipid droplets, many papers nevertheless report the use of higher doses of dex for the osteogenic differentiation of hBMSCs. A recent paper also deals with the effect of different concentration of BMSCs in osteogenic and adipogenic differentiation [40]. In that paper, it was shown that the 100-nM dose of dex resulted in a higher level of mineralization in hBMSC osteogenic cultures, suggesting this concentration for the induction of osteogenesis. However, our results so far suggest that a lower concentration of dex $(10 \mathrm{nM})$ might be better to maximize its pro-osteogenic effects, with higher levels of late marker expression (i.e., BGLAP and IBSP), lower expression of adiponectin, and an overall minor presence of adipocyte-like cells.

The double-edged effect of dex on differentiation has been demonstrated before in murine cells, where it was indeed observed that dex may lead to heterogeneous osteogenic/adipogenic commitment [27]. Notably, it was also suggested that osteoblasts with high PPARG expression may switch fate and become adipocytes [28]. In addition, our results are in line with previous reports showing that dex promotes adipose tissue to the detriment of bone formation in bone marrow [10].

Our results also indicate that dex is required to induce downregulation of SOX9, and it counteracts the effect of ascorbic acid and glycerol 2-phosphate alone, which conversely upregulated $S O X 9$ expression. $S O X 9$ downregulation by dex has been previously reported in cells from the brain system (murine astrocytes [41,42] and neural stem cells [41,43] with $100 \mathrm{nM}$ dex, and in rat hypothalamus [41,44] with a $2 \mathrm{mg} / \mathrm{kg}$ dose in vivo) and in the mouse chondroprogenitor cell line ATDC5 $(1 \mu \mathrm{M})$ [45].

The balance of three key transcription factors for the determination of cell lineage (i.e., RUNX2 for osteogenic [19], SOX9 for chondrogenic [46], and PPARG for adipogenic differentiation [47]) is pivotal in addressing which fate the cell will undertake. Our group has previously shown that the RUNX2/SOX9 ratio, rather than RUNX2 alone, is a better predictor of the mineralization potential of hBMSCs due to changes in SOX9 expression [31]. Here we propose a model which also takes PPARG into consideration and shows how its expression is dependent on dex treatment. It is worth highlighting that the effects pointed out by the $\mathrm{ABC}$ simulations are in support of a role for SOX9 that counters the indirect stimulatory effect of dex. According to those simulations, the signal that passes through SOX9 to RUNX2, and consequently stimulates bone formation, relies on the strong SOX9 feedback and its slow degradation rate, which both act disruptively for the disinhibition pathway of dex $\dashv$ SOX $9 \dashv R U N X 2$ (where $\dashv$ represents the inhibition symbol). This is also suggested by the stronger inhibitory interaction of SOX9 with RUNX2, compared to the inverse effect of RUNX2 $\dashv S O X 9$. On one hand, this $A B C$ model successfully captured the experimental trends of gene expression. On the other hand, the range of the responses were different, which might indicate that the model scheme used to carry out the simulations was oversimplified, and that further signaling components are necessary for a better convergence of the model with the experimental observations.

It is worth mentioning that the present work is relevant for understanding mechanisms underlying intramembranous ossification. Indeed, with this in vitro model, we targeted the direct differentiation of hBMSCs to the osteoblastic lineage. From our results, we cannot infer directly what the effect of dex is during endochondral ossification and how the transcription factors are regulated, as the two processes and the in vitro models are very different. However, exogenous glucocorticoid administration is known to induce long bone growth retardation, probably through several direct and indirect mechanisms that affect chondrocytes and vascular invasion [48]. Dexamethasone is also a common component of chondrogenic differentiation cocktails; intriguingly, we have previously shown that its absence ameliorates the chondrogenic differentiation of synovial derived stem cells induced by TGF- $\beta 1$ and BMP-2, whereas its presence usually results in higher PPARG levels [49].

Further investigation is warranted to better understand the effect of dex on a single-cell level. Indeed, hBMSC cultures comprise a heterogeneous population of cells with different degrees of potential and differentiation. Usually, it is supposed that the relative activity of 
RUNX2 versus PPARG decides the cell fate commitment toward osteo- or adipogenesis, which are mutually exclusive [50]. Since in our cultures we could observe both types of differentiation at the same time, it is likely that there might be a different response to dex at a single-cell level. Whether dex selects for cells with specific characteristics (e.g., physiologically less osteogenic and with a more adipogenic-committed phenotype) or not is crucial to better understand how dex controls differentiation. Moreover, this may lead us toward understanding how to maintain only the desirable effects of dex (which is, SOX9 downregulation and osteogenic differentiation), while excluding the activation of off-target pathways leading to adipogenic commitment. This would be fundamental for a proper clinical translation of in vitro osteogenesis results. Another point that requires further studies is identifying which mediators lead to SOX9 downregulation. Indeed, our results suggest that this effect is mediated mainly by a transactivation pathway, since we could not observe comparable results by treating cells with the same concentrations of (+)-ZK 216348. Therefore, we should aim to understand whether it is PPARG that directly induces SOX9 downregulation alone, or if there is any other actor involved. If the former hypothesis is true, this would mean that the adipogenic and the osteogenic effects of dex are more closely intertwined than expected; therefore, completely new approaches to in vitro osteogenic differentiation should be developed. Finally, our results indicate that there is a high donor-related variation of RUNX2 expression in response to dex. Clarifying this aspect might be crucial in order to better predict the differentiation potential of hBMSCs. In the present work, age, gender, and cell duplication time in culture did not seem to predict the response of RUNX2 expression to dexamethasone (data not shown).

In conclusion, here we show that despite its role in inducing osteogenic differentiation via the inhibition of SOX9 expression, the effects of dex are too broad and an adipogenic program can also be initiated, in at least a subset of cells. We need to rethink how we study differentiation in vitro, with the commonly used standard protocols not necessarily representing the best solution. One alternative might involve the use of two- or multistage protocols, with each stage being more adapted to the differentiation stage (induction $\neq$ mineralization and maturation). In this work, we have tested a two-stage protocol that is still based on the use of dexamethasone, in which we were able to show a slight improvement. A short burst of dexamethasone in the early differentiation commitment is probably more beneficial for the cells, but our hypothesis is that we should avoid dexamethasone completely if we want to improve research in the bone field. Indeed, on one side we show that we can limit the exposure of cells to dex and still achieve good levels of mineralization, and this approach might be further improved by focusing on even earlier time points. On the other hand, this work raises the urgent need to find new strategies and compounds that can induce SOX9 downregulation, while excluding PPARG upregulation.

\section{Materials and Methods}

\subsection{Cell Isolation and Induction of Osteogenic Differentiation}

Isolation of mesenchymal stromal cells from human bone marrow (hBMSCs) was performed as described before [16]. After obtaining full ethical approval (KEK-ZH:20100444/0) and with written informed consent, cells from 15 donors were utilized (9 M/6 F; mean age 42 years; age range 15-80; see Figure 54 ). After isolation, an initial cell density of $3 \times 10^{3}$ cells $/ \mathrm{cm}^{2}$ was maintained for the hBMSC subculture, and these were grown until passage 2 (p2) in Minimum Essential Medium Eagle-Alpha Modification ( $\alpha$-MEM, Gibco, Thermo Fisher, Zürich, Switzerland) with the addition of $100 \mu \mathrm{g} / \mathrm{mL}$ streptomycin (Gibco), $100 \mathrm{U} / \mathrm{mL}$ penicillin (Gibco), $5 \mathrm{ng} / \mathrm{mL}$ basic fibroblast growth factor (bFGF, Fitzgerald Industries International, Acton, MA, USA), and 10\% MSC-qualified fetal bovine serum (FBS, Pan-Biotech, Aidenbach, Germany). Maintenance of cultures was performed at $37^{\circ} \mathrm{C} / 5 \% \mathrm{CO}_{2}$, and the medium was refreshed on alternate days.

For osteogenic differentiation, seeding of cells at $\mathrm{p} 3$ was performed at a density of $1.5 \times 10^{4}$ cells $/ \mathrm{cm}^{2}$ on Thermanox coverslips (Nunc, Milian AG, Geneve, Switzerland). The medium was changed after overnight cell attachment; cultures of control (CRL) samples 
was carried out in Dulbecco's modified Eagle's medium (DMEM) with $1 \mathrm{~g} / \mathrm{L}$ glucose, $100 \mathrm{\mu g} / \mathrm{mL}$ streptomycin, $100 \mathrm{U} / \mathrm{mL}$ penicillin, and $10 \%$ heat-inactivated FBS (all from Gibco). The addition of $5 \mathrm{mM} \beta$-glycerol phosphate, $50 \mu \mathrm{g} / \mathrm{mL}$ ascorbic acid 2-phosphate, and varying concentrations of dexamethasone-cyclodextrin complex (water-soluble formulation) to the control medium was used to induce osteogenic differentiation (all from Sigma-Aldrich, Buchs, Switzerland). Cells were maintained in osteogenic medium with different dex concentrations $(0 \mathrm{nM}, 10 \mathrm{nM}$, and $100 \mathrm{nM})$ for 21 days ( $\mathrm{n}=3$ donors) or with two-stage protocols, as described in Table 1 ( $\mathrm{n}=4$ donors). To maintain stable levels of differentiation factors, the medium was refreshed on alternate days. For RNA isolation, the collection of samples was performed at day 7 and day 21. Furthermore, for evaluating final differentiation outcomes, Alizarin Red S and Oil Red O staining were performed on day 21 to evidence calcium deposition and the presence of lipid droplets, respectively. In a separate experiment ( $\mathrm{n}=8$ donors), the same concentrations of dex, (+)-ZK 216348 (Axon Medchem BV, Groningen, The Netherlands), or dimethyl sulfoxide (DMSO) as a vehicle control were tested in osteogenic differentiation, and RNA was isolated after 7 days for gene expression analysis.

\subsection{Alizarin Red Staining and Quantification}

Samples at day 21 were fixed in $4 \%$ neutral buffered formalin and stained with a $40 \mathrm{mM}, \mathrm{pH} 4.2$ solution of Alizarin Red S (Sigma-Aldrich). After extensive washing to remove unbound staining, mosaic pictures were created with an Evos2 microscope (ThermoFisher). Subsequently, Alizarin Red was eluted from cultures, using the cetylpyridinium chloride method, as previously described [51], and quantification was performed by measuring absorbance at $540 \mathrm{~nm}$.

\subsection{Oil Red O Staining and Quantification}

Samples at day 21 were fixed in $4 \%$ neutral buffered formalin, washed with 2-propanol, and stained with an Oil Red O working solution (0.5\% Oil Red O in 2-propanol, $60 \%$ in $\mathrm{H}_{2} \mathrm{O}$ ). After a further wash in 2-propanol, the cultures were washed and maintained in $\mathrm{H}_{2} \mathrm{O}$ for imaging. Pictures were acquired using an Axiovert 40 inverted microscope, CPAchromat $5 \times / 0.12$ objective, equipped with an Axiocam 105 color camera (Carl Zeiss Microscopy GmbH, Jena, Germany). At least 10 representative pictures were taken for further image analysis. Images were analyzed with Fiji/ImageJ software (NIH, Bethesda, MD, USA). Briefly, raw images were imported and converted to RGB. A color threshold was applied to select stained cells, then the area covered by the selection was measured (in $\mu \mathrm{m}^{2}$ ). The percentage out of the total area was calculated. Final results were calculated as the average $\%$ area of the 10 images.

\subsection{Isolation of $R N A$}

Standard TRIreagent extraction (Molecular Research Center Inc., Cincinnati, OH, USA) with 1-bromo-3-chloropropane (Sigma-Aldrich) was used for the isolation of total RNA. After phase separation, the precipitation of RNA was carried out from the aqueous phase by adding 2-propanol (Sigma-Aldrich). The RNA pellet was washed with $75 \% \mathrm{EtOH}$ and finally reconstituted with diethylpyrocarbonate (DEPC)-treated $\mathrm{H}_{2} \mathrm{O}$. A NanoDrop 1000 (Thermo Fisher) was used to measure the total RNA concentration and purity was evaluated via assessment of the A260/280 and A260/230 ratios.

\subsection{Gene Expression Analysis}

For total gene expression, TaqMan Reverse Transcription reagents (Applied Biosystems, Foster City, CA, USA) were used to synthesize cDNA from 1000 ng of total RNA, as per the recommendations of the manufacturer. Differentiation markers in osteogenic differentiation (see Table S1) were analyzed using qPCR. TaqMan Gene Expression Master Mix (Applied Biosystems) in a QuantStudio 7 Flex Real-Time PCR system (Applied Biosystems) was used to achieve the amplification of target genes by applying the following protocol: 
$2 \mathrm{~min}$ at $50{ }^{\circ} \mathrm{C} ; 10 \mathrm{~min}$ at $95^{\circ} \mathrm{C} ; 40$ cycles of $15 \mathrm{sec}$ at $95^{\circ} \mathrm{C}, 1 \mathrm{~min}$ at $60^{\circ} \mathrm{C}$. TaqMan gene expression assay (Thermo Fisher) or custom-designed primers and probes (Microsynth AG, Balgach, Switzerland) were used to test the genes of interest (Table S1). Results were expressed as $2^{-\Delta \mathrm{Ct}}$, with $R P L P 0$ used as a reference gene.

\subsection{Approximate Bayesian Computation Simulations}

A network interaction model, including four molecular species, one activation, seven inhibitory, and three positive feedback reactions, was used as an input to carry out steadystate simulations [36,52]. The method used basal/decay rates and Hill-type transfer functions for species and reactions, respectively, and solved ordinary differential equations that determined the dynamics of the system at the steady state, to be compared to the results obtained experimentally [37]. Interactions between species were assumed to be linear by making the Hill coefficient of each reaction equal to one, as for the other model parameters, unless otherwise specified.

Independently, the basal activities of RUNX2, SOX9, and PPARG were kept as fixed parameters in the simulations, at the values measured experimentally corresponding to $0 \mathrm{nM}$ dex stimulation. Dex activity was used as a corresponding measure of the experimental concentrations, by means of scaling experimental and simulated data (both were scaled to 1 by dividing by their respective maximal value). All other parameters that determine the decay of RUNX2, SOX9, and PPARG, as well as the affinities of their interactions, including the strength of the positive feedback loops, were varied according to a random scheme. The range of decay rates was [0.001-1], whereas the range of affinities was [0.001-100]. The steady-state values generated by the model according to this sampling scheme constituted the prior probability distribution used for ABC (Figure S1).

Full data were used instead of summary statistics to carry out ABC using the Euler distance method between the experimental data per each dex concentration, and the steady state of the simulated data. The CRAN ABC package [53] was used, with a tolerance of 0.005, using the rejection method to infer the posterior probability distribution (Figure S2) and consequently to estimate model parameters that best represented the experimental results.

\subsection{Heatmap Generation and Statistical Analysis}

Differential expression levels of genes were calculated as Log2 (ratios) of hBMSCs treated with pro-osteogenic medium +/- dex at different concentrations (10 nM, $100 \mathrm{nM})$, normalized to their respective untreated condition (control = CTRL). Genes with a Log2FC $\geq 0.585$ (corresponding to a 1.5 -fold change) were considered to be differentially expressed. Hierarchical clustering on donors and colorimetric representation of the relative transcriptional profiles were represented through heat-maps generated by Heatmapper [54] (https:/ / github.com/WishartLab/heatmapper, accessed on 19 March 2021), setting the Pearson correlation as the distance measure and average linkage as the clustering method.

Statistical analysis was performed using GraphPad Prism v.8 (GraphPad Software, San Diego, CA, USA) for the analysis of gene expression with increasing doses of dex (or (+)-ZK 216348). After testing for a Gaussian normal distribution with the Shapiro-Wilk normality test, a repeated-measure one- or two- way Analysis of Variance (ANOVA) with the GeisserGreenhouse correction was applied, followed by Tukey's multiple comparison test to compare the means between the different groups or by testing for linear trends. Matching was effective for the genes tested, with a $p=0.0002$ for RUNX2, $p<0.0001$ for SOX9, and $p=0.0020$ for PPARG. Pearson's coefficient was calculated to test possible correlations. 
Supplementary Materials: The following are available online at https: / www.mdpi.com/article/ 10.3390/ijms22094785/s1, Figure S1: Prior (uniformly random) distributions. Figure S2: Posterior (selected by ABC) distributions. Figure S3: Gene expression profiling and donor clustering analysis of RUNX2 in response to pro-osteogenic medium with different concentrations of dex $(0 \mathrm{nM}, 10 \mathrm{nM}$, $100 \mathrm{nM}$ ) in isolated hBMSCs. Figure S4: Summary of BMSC donors used in each experiment. Table S1: List and details of primers and probes used for gene expression analysis. Table S2: complete dataset of Log2FC values of RUNX2, SOX9, and PPARG expression.

Author Contributions: Conceptualization, E.D.B and M.J.S.; methodology, E.D.B. and M.J.S.; investigation, E.D.B. and P.A.; validation, P.A.; formal analysis, E.D.B., A.B.-D. and G.L.; writing-original draft preparation, E.D.B., A.B.-D., G.L. and P.A.; writing-review and editing, E.D.B., V.B., M.A. and M.J.S.; visualization, E.D.B., A.B.-D. and G.L.; supervision, M.J.S.; project administration, M.J.S.; funding acquisition, M.A. and M.J.S. All authors have read and agreed to the published version of the manuscript.

Funding: This investigation was supported by AO Foundation and AOTrauma.

Institutional Review Board Statement: The study was conducted according to the guidelines of the Declaration of Helsinki and approved by the Institutional Review Board (or Ethics Committee) of Zurich Cantonal Authorities (KEK-ZH:2010-0444/0).

Informed Consent Statement: Informed consent was obtained from all subjects involved in the study.

Data Availability Statement: All data and materials used in the analysis are available to any researcher for purposes of reproducing or extending the analysis.

Conflicts of Interest: The authors declare no conflict of interest.

\section{References}

1. Van Heest, A.; Swiontkowski, M. Bone-graft substitutes. Lancet 1999, 353 (Suppl. 1), SI28-SI29. [CrossRef]

2. Giannoudis, P.V.; Dinopoulos, H.; Tsiridis, E. Bone substitutes: An update. Injury 2005, 36 (Suppl. 3), S20-S27. [CrossRef] [PubMed]

3. Hulsart-Billstrom, G.; Dawson, J.I.; Hofmann, S.; Muller, R.; Stoddart, M.J.; Alini, M.; Redl, H.; El Haj, A.; Brown, R.; Salih, V.; et al. A surprisingly poor correlation between in vitro and in vivo testing of biomaterials for bone regeneration: Results of a multicentre analysis. Eur. Cell Mater. 2016, 31, 312-322. [CrossRef]

4. Barnes, P.J.; Adcock, I.M. Glucocorticoid resistance in inflammatory diseases. Lancet 2009, 373, 1905-1917. [CrossRef]

5. Wang, Y.; Zhao, R.; Gu, Z.; Dong, C.; Guo, G.; Li, L. Effects of glucocorticoids on osteoporosis in rheumatoid arthritis: A systematic review and meta-analysis. Osteoporos. Int. 2020, 31, 1-9. [CrossRef] [PubMed]

6. Buckley, L.; Humphrey, M.B. Glucocorticoid-Induced Osteoporosis. N. Engl. J. Med. 2018, 379, 2547-2556. [CrossRef]

7. Osmani, F.; Thakkar, S.; Vigdorchik, J. The utility of conservative treatment modalities in the management of osteonecrosis: A systematic review. Bull. NYU Hosp. Jt. Dis. 2017, 75, 186.

8. Weinstein, R.S. Glucocorticoid-induced osteonecrosis. Endocrine 2012, 41, 183-190. [CrossRef]

9. Eijken, M.; Koedam, M.; van Driel, M.; Buurman, C.J.; Pols, H.A.; van Leeuwen, J.P. The essential role of glucocorticoids for proper human osteoblast differentiation and matrix mineralization. Mol. Cell. Endocrinol. 2006, 248, 87-93. [CrossRef]

10. Li, J.; Zhang, N.; Huang, X.; Xu, J.; Fernandes, J.C.; Dai, K.; Zhang, X. Dexamethasone shifts bone marrow stromal cells from osteoblasts to adipocytes by C/EBPalpha promoter methylation. Cell Death Dis. 2013, 4, e832. [CrossRef]

11. Henneicke, H.; Gasparini, S.J.; Brennan-Speranza, T.C.; Zhou, H.; Seibel, M.J. Glucocorticoids and bone: Local effects and systemic implications. Trends Endocrinol. Metabolism. 2014, 25, 197-211. [CrossRef]

12. Zhou, H.; Cooper, M.S.; Seibel, M.J. Endogenous glucocorticoids and bone. Bone Res. 2013, 1, 107-119. [CrossRef] [PubMed]

13. Haas, A.V.; LeBoff, M.S. Osteoanabolic agents for osteoporosis. J. Endocr. Soc. 2018, 2, 922-932. [CrossRef] [PubMed]

14. Cheng, S.L.; Yang, J.W.; Rifas, L.; Zhang, S.F.; Avioli, L.V. Differentiation of human bone marrow osteogenic stromal cells in vitro: Induction of the osteoblast phenotype by dexamethasone. Endocrinology 1994, 134, 277-286. [CrossRef] [PubMed]

15. Langenbach, F.; Handschel, J. Effects of dexamethasone, ascorbic acid and beta-glycerophosphate on the osteogenic differentiation of stem cells in vitro. Stem Cell Res. Ther. 2013, 4, 117. [CrossRef] [PubMed]

16. Gardner, O.F.W.; Alini, M.; Stoddart, M.J. Mesenchymal Stem Cells Derived from Human Bone Marrow. In Cartilage Tissue Engineering: Methods and Protocols; Doran, P.M., Ed.; Springer: New York, NY, USA, 2015. [CrossRef]

17. Hanna, H.; Mir, L.M.; Andre, F.M. In vitro osteoblastic differentiation of mesenchymal stem cells generates cell layers with distinct properties. Stem Cell Res. Ther. 2018, 9, 203. [CrossRef]

18. Hamidouche, Z.; Hay, E.; Vaudin, P.; Charbord, P.; Schule, R.; Marie, P.J.; Fromigue, O. FHL2 mediates dexamethasone-induced mesenchymal cell differentiation into osteoblasts by activating Wnt/beta-catenin signaling-dependent Runx2 expression. FASEB J. 2008, 22, 3813-3822. [CrossRef] 
19. Bruderer, M.; Richards, R.G.; Alini, M.; Stoddart, M.J. Role and regulation of RUNX2 in osteogenesis. Eur. Cell Mater. 2014, 28, 269-286. [CrossRef]

20. Felthaus, O.; Gosau, M.; Klein, S.; Prantl, L.; Reichert, T.E.; Schmalz, G.; Morsczeck, C. Dexamethasone-related osteogenic differentiation of dental follicle cells depends on ZBTB16 but not Runx2. Cell Tissue Res. 2014, 357, 695-705. [CrossRef]

21. Maioli, M.; Basoli, V.; Santaniello, S.; Cruciani, S.; Delitala, A.P.; Pinna, R.; Milia, E.; Grillari-Voglauer, R.; Fontani, V.; Rinaldi, S.; et al. Osteogenesis from Dental Pulp Derived Stem Cells: A Novel Conditioned Medium Including Melatonin within a Mixture of Hyaluronic, Butyric, and Retinoic Acids. Stem Cells Int. 2016, 2016, 2056416. [CrossRef]

22. Alm, J.J.; Heino, T.J.; Hentunen, T.A.; Vaananen, H.K.; Aro, H.T. Transient 100 nM dexamethasone treatment reduces inter- and intraindividual variations in osteoblastic differentiation of bone marrow-derived human mesenchymal stem cells. Tissue Eng. Part C Methods 2012, 18, 658-666. [CrossRef] [PubMed]

23. Wong, M.M.; Rao, L.G.; Ly, H.; Hamilton, L.; Tong, J.; Sturtridge, W.; McBroom, R.; Aubin, J.E.; Murray, T.M. Long-term effects of physiologic concentrations of dexamethasone on human bone-derived cells. J. Bone Miner. Res. 1990, 5, 803-813. [CrossRef]

24. Rimando, M.G.; Wu, H.H.; Liu, Y.A.; Lee, C.W.; Kuo, S.W.; Lo, Y.P.; Tseng, K.F.; Liu, Y.S.; Lee, O.K. Glucocorticoid receptor and Histone deacetylase 6 mediate the differential effect of dexamethasone during osteogenesis of mesenchymal stromal cells (MSCs). Sci. Rep. 2016, 6, 37371. [CrossRef] [PubMed]

25. Leclerc, N.; Noh, T.; Khokhar, A.; Smith, E.; Frenkel, B. Glucocorticoids inhibit osteocalcin transcription in osteoblasts by suppressing Egr2/Krox20-binding enhancer. Arthritis Rheum. 2005, 52, 929-939. [CrossRef]

26. Della Bella, E.; Menzel, U.; Basoli, V.; Tourbier, C.; Alini, M.; Stoddart, M.J. Differential Regulation of circRNA, miRNA, and piRNA during Early Osteogenic and Chondrogenic Differentiation of Human Mesenchymal Stromal Cells. Cells 2020, 9, 398. [CrossRef] [PubMed]

27. Ghali, O.; Broux, O.; Falgayrac, G.; Haren, N.; van Leeuwen, J.P.; Penel, G.; Hardouin, P.; Chauveau, C. Dexamethasone in osteogenic medium strongly induces adipocyte differentiation of mouse bone marrow stromal cells and increases osteoblast differentiation. BMC Cell Biol. 2015, 16, 9. [CrossRef]

28. Yoshiko, Y.; Oizumi, K.; Hasegawa, T.; Minamizaki, T.; Tanne, K.; Maeda, N.; Aubin, J.E. A subset of osteoblasts expressing high endogenous levels of PPARgamma switches fate to adipocytes in the rat calvaria cell culture model. PLoS ONE 2010, 5, e11782. [CrossRef]

29. Frith, J.; Genever, P. Transcriptional control of mesenchymal stem cell differentiation. Transfus. Med. Hemother. 2008, 35, $216-227$. [CrossRef]

30. van de Peppel, J.; Strini, T.; Tilburg, J.; Westerhoff, H.; van Wijnen, A.J.; van Leeuwen, J.P. Identification of Three Early Phases of Cell-Fate Determination during Osteogenic and Adipogenic Differentiation by Transcription Factor Dynamics. Stem Cell Rep. 2017, 8, 947-960. [CrossRef]

31. Loebel, C.; Czekanska, E.M.; Bruderer, M.; Salzmann, G.; Alini, M.; Stoddart, M.J. In vitro osteogenic potential of human mesenchymal stem cells is predicted by Runx2/Sox9 ratio. Tissue Eng. Part A 2015, 21, 115-123. [CrossRef]

32. Schacke, H.; Schottelius, A.; Docke, W.D.; Strehlke, P.; Jaroch, S.; Schmees, N.; Rehwinkel, H.; Hennekes, H.; Asadullah, K. Dissociation of transactivation from transrepression by a selective glucocorticoid receptor agonist leads to separation of therapeutic effects from side effects. Proc. Natl. Acad. Sci. USA 2004, 101, 227-232. [CrossRef]

33. MacArthur, B.D.; Please, C.P.; Oreffo, R.O. Stochasticity and the molecular mechanisms of induced pluripotency. PLoS ONE 2008, 3, e3086. [CrossRef] [PubMed]

34. Buetti-Dinh, A.; Friedman, R. Computer simulations of the signalling network in FLT3 (+)-acute myeloid leukaemia-Indications for an optimal dosage of inhibitors against FLT3 and CDK6. BMC Bioinform. 2018, 19, 155. [CrossRef]

35. Buetti-Dinh, A.; O'Hare, T.; Friedman, R. Sensitivity Analysis of the NPM-ALK Signalling Network Reveals Important Pathways for Anaplastic Large Cell Lymphoma Combination Therapy. PLoS ONE 2016, 11, e0163011. [CrossRef] [PubMed]

36. Buetti-Dinh, A.; Pivkin, I.V.; Friedman, R. S100A4 and its role in metastasis-computational integration of data on biological networks. Mol. Biosyst. 2015, 11, 2238-2246. [CrossRef]

37. Buetti-Dinh, A.; Herold, M.; Christel, S.; El Hajjami, M.; Delogu, F.; Ilie, O.; Bellenberg, S.; Wilmes, P.; Poetsch, A.; Sand, W.; et al. Reverse engineering directed gene regulatory networks from transcriptomics and proteomics data of biomining bacterial communities with approximate Bayesian computation and steady-state signalling simulations. BMC Bioinform. 2020, 21, 23. [CrossRef]

38. Hoffman, T.; Khademhosseini, A.; Langer, R. Chasing the Paradigm: Clinical Translation of 25 Years of Tissue Engineering. Tissue Eng. Part A 2019, 25, 679-687. [CrossRef] [PubMed]

39. Weinstein, R.S. Glucocorticoids, osteocytes, and skeletal fragility: The role of bone vascularity. Bone 2010, 46, 564-570. [CrossRef]

40. Li, T.; Xu, Y.; Wang, Y.; Jiang, Y. Differential expression profiles of long noncoding RNAs and mRNAs in human bone marrow mesenchymal stem cells after exposure to a high dosage of dexamethasone. Stem Cell Res. Ther. 2021, 12, 9. [CrossRef]

41. Juszczak, G.R.; Stankiewicz, A.M. Glucocorticoids, genes and brain function. Prog. Neuropsychopharmacol. Biol. Psychiatry 2018, 82, 136-168. [CrossRef]

42. Carter, B.S.; Meng, F.; Thompson, R.C. Glucocorticoid treatment of astrocytes results in temporally dynamic transcriptome regulation and astrocyte-enriched mRNA changes in vitro. Physiol. Genom. 2012, 44, 1188-1200. [CrossRef] [PubMed] 
43. Peffer, M.E.; Chandran, U.R.; Luthra, S.; Volonte, D.; Galbiati, F.; Garabedian, M.J.; Monaghan, A.P.; DeFranco, D.B. Caveolin-1 regulates genomic action of the glucocorticoid receptor in neural stem cells. Mol. Cell. Biol. 2014, 34, 2611-2623. [CrossRef] [PubMed]

44. Sato, H.; Horikawa, Y.; Iizuka, K.; Sakurai, N.; Tanaka, T.; Shihara, N.; Oshima, A.; Takeda, J.; Mikuni, M. Large-scale analysis of glucocorticoid target genes in rat hypothalamus. J. Neurochem. 2008, 106, 805-814. [CrossRef]

45. Naito, M.; Ohashi, A.; Takahashi, T. Dexamethasone inhibits chondrocyte differentiation by suppression of Wnt/beta-catenin signaling in the chondrogenic cell line ATDC5. Histochem. Cell Biol. 2015, 144, 261-272. [CrossRef]

46. Akiyama, H.; Chaboissier, M.C.; Martin, J.F.; Schedl, A.; de Crombrugghe, B. The transcription factor Sox9 has essential roles in successive steps of the chondrocyte differentiation pathway and is required for expression of Sox 5 and Sox6. Genes Dev. 2002, 16, 2813-2828. [CrossRef]

47. Chen, Q.; Shou, P.; Zheng, C.; Jiang, M.; Cao, G.; Yang, Q.; Cao, J.; Xie, N.; Velletri, T.; Zhang, X.; et al. Fate decision of mesenchymal stem cells: Adipocytes or osteoblasts? Cell Death Differ. 2016, 23, 1128-1139. [CrossRef]

48. Hartmann, K.; Koenen, M.; Schauer, S.; Wittig-Blaich, S.; Ahmad, M.; Baschant, U.; Tuckermann, J.P. Molecular Actions of Glucocorticoids in Cartilage and Bone During Health, Disease, and Steroid Therapy. Physiol. Rev. 2016, 96, 409-447. [CrossRef]

49. Kovermann, N.J.; Basoli, V.; Della Bella, E.; Alini, M.; Lischer, C.; Schmal, H.; Kubosch, E.J.; Stoddart, M.J. BMP2 and TGF-beta Cooperate Differently during Synovial-Derived Stem-Cell Chondrogenesis in a Dexamethasone-Dependent Manner. Cells 2019, 8, 636. [CrossRef]

50. Ge, C.; Cawthorn, W.P.; Li, Y.; Zhao, G.; Macdougald, O.A.; Franceschi, R.T. Reciprocal Control of Osteogenic and Adipogenic Differentiation by ERK/MAP Kinase Phosphorylation of Runx2 and PPARgamma Transcription Factors. J. Cell. Physiol. 2016, 231, 587-596. [CrossRef]

51. Della Bella, E.; Pagani, S.; Giavaresi, G.; Capelli, I.; Comai, G.; Donadei, C.; Cappuccilli, M.; La Manna, G.; Fini, M. Uremic Serum Impairs Osteogenic Differentiation of Human Bone Marrow Mesenchymal Stromal Cells. J. Cell. Physiol. 2017, 232, 2201-2209. [CrossRef]

52. Buetti-Dinh, A.; Pivkin, I.V.; Friedman, R. S100A4 and its role in metastasis—Simulations of knockout and amplification of epithelial growth factor receptor and matrix metalloproteinases. Mol. Biosyst. 2015, 11, 2247-2254. [CrossRef] [PubMed]

53. Csilléry, K.; François, O.; Blum, M.G.B. abc: An R package for approximate Bayesian computation (ABC). Methods Ecol. Evol. 2012, 3, 475-479. [CrossRef]

54. Babicki, S.; Arndt, D.; Marcu, A.; Liang, Y.; Grant, J.R.; Maciejewski, A.; Wishart, D.S. Heatmapper: Web-enabled heat mapping for all. Nucleic Acids Res. 2016, 44, W147-W153. [CrossRef] [PubMed] 